\title{
Sulle successioni di funzioni quasi continue negli spazi astratti ( $\left.{ }^{1}\right)$.
}

\author{
di Tuaro Vrora (a Bari).
}

A Maura Picone nel suo $70^{\text {no }}$ compleanno.

Sunto. - Si studia una definizione del tutto generale di funzione quasi continua; si enuncia e dimostra poi un teorema, concettualmente nuovo, per le successioni di funzioni che sono quasi continue nel senso generale studiato.

1. Il concetto di funzione quasi continua, introdotto nell'analisi matematica e largamente usato da L. ToNELLI, è stato generalizzato, com'è noto, da M. Proone e dalla Sua scuola, rivelando tutta la sua importanza in diversi argomenti fondamentali, principalmente nella teoria dell'integrazione $\left({ }^{*}\right)$.

Recentemente G. Frchers $\left({ }^{3}\right)$ ha persino dato il concetto molto generale di funzione reale quasi continua in un insieme misurabile di un qualunque spazio topologico S. La misurabilità è intesa, da FICHERA, con riferimento ad una misura o funzione reale $\mu(e)$ definita nella famiglia $\mathscr{F}$ degli insiemi boreliani $e$ di $S$; la funzione $\mu(e)$ è supposta completamente additiva ed a variazione finita. In conseguenza, anche la quasi continuità $\partial$ intesa con riferimento alla stessa misura $\mu(e)$.

La definizione di funzione $f(P)$ quasi continua può darsi in ipotesi ancora più generali, quando si supponga: che $\mu(e)$ assuma valori (") appartenenti a uno spazio $S^{\prime}$ soddisfacente ad opportune condizioni di additività, ed $f(P)$ valori appartenenti ad uno spazio topologico $S^{\prime \prime}$. In questo lavoro mostrerò come una tale generalissima definizione si adatti a buona parte della teoria svolta da Prcone senza sostanziali cambiamenti, ove si aggiunga allo spazio $S$ la condizione d'esser metrico e separabile (5). Se poi si suppone, più precisa nente, che $S^{\prime \prime}$ sia metrico e completo, si può molto facilmente

(1) Lavoro eseguito presso l'Istituto Nazionale per le Applicazioni del Calcolo.

(2) Ofr. M. PiCONe e T. VIolA, Lezioni sulla teoria moderna dell'integrazione (Torino, Einaudi, 1952) pp. 124 e segg. Questo trattato verrà considerato, nel seguito del presente lavoro, come fondamentale e richiamato semplicemente con la sigla $P V$.

(3) G. Frohera, Lezioni sulle trasformazioni lineari, vol. I (Trieste, pr. l'Istituto Matematico dell' Università, 1954) pp. 327.330.

(4) La parola * valore " $\dot{~ q u i ~ i n t e s a ~ c o m e ~ s i n o n i m o ~ d e l l a ~ p a r o l a ~ s ~ p u n t o ~ * . ~}$

(5) La separabilità avrà, in sostanza, lo seopo di regolarizzare i procedimenti costruttivi, nel senso di liberarli dal postulato delle infinite scelte arbitrarie. 
pervenire anche qui al teorema fondamentale (da considerarsi come un corollario di quello di SeverinI-EgOROFF $\left.\left({ }^{6}\right)\right)$ : se una successione

$$
f_{\mathrm{i}}(P), f_{\mathrm{\varepsilon}}(P), \ldots, f_{n}(P), \ldots
$$

di funzioni quasi continue in un insieme lebesghiano $I$, converge quasi ovunque in $I$, la funzione $f(P)$ cui essa tende è anch'essa quasi continua in $I$.

2. Ma lo scopo principale del presente lavoro non si riduce ad una generalizzazione che potrebbe, forse, non apparire, in sè, nè imprevedibile nè difficile $\left({ }^{7}\right)$ : Qui, volendo fare opera non indegna dell'illustre Maestro, mi propongo principalmente di dare la dimostrazione d'un teorema che, a quanto mi risulta, è da ritenersi del tutto nuovo. Introducendo ulteriormente, in via pregiudiziale, la condizione restrittiva per $S^{\prime \prime} d$ 'esser compatto e separabile, il nuovo teorema (nel quale quello fondamentale, ricordato alla fine del n. 1, rientra come caso particolarissimo) è suscettibile del seguente enunciato:

se $\left\{f_{n}(P)\right\}$ è una successione di funzioni tutte quasi continue in uno stesso insieme $I$, ̀̀ possibile costruire una funzione $f(P)$ quasi continua in $I$ che, in quasi tutti $i$ punti $P$ di $I$, è uguale a uno dei valori limiti della successione.

\section{I fondamenti della teoria.}

3. Quanto precede non assume an significato pienamente determinato, finchè non si enuncino esplicitamente le proprietà dello spazio $S^{\prime}$ di cui sopra. Ritengo che, volendo richiedere ad $S^{\prime}$ un minimo possibile di restrizioni, non si possa tralasciare alcuna delle proprietà già imposte da M. FrécHeT nella sua fondamentale memoria: L'intégrale abstraite d'une fonction abstraite d' une variable abstraite et son application à la moyenne d'un élément aléatoire de nature quelconque (Revue Scientifique, vol. 82, dic. 1944, pp. 483.512). Mi limito perciò a richiamare succintamente quelle proprietà (ivi p. 503).

Se $v^{\prime}, v^{\prime \prime}$ sono due punti qualunque di $S^{\prime}$, esiste in $S^{\prime}$ la somma $v=v^{\prime}+v^{\prime \prime}$ unica $\theta$ ben determinata, per la quale valgono le proprietà commutativa ed associativa.

L' equazione $\gamma^{\prime}=\gamma^{\prime \prime}+\gamma$ nell' incognita $\gamma$, ̀̀ sempre univocamente risolubile: la sua soluzione $\gamma$ è la differenza $v^{\prime}-v^{\prime \prime}$. Si deduce immediatamente l'esistenza dello zero $0=v-v$, indipendente da $\vee$.

(8) Cfr. PV, p. 139.

(7) Mi propongo tuttavia di riprendere, in altra sede, l'argomento per dimostrarne l'interesse in questioni attinenti alla teoria dell' integrazione. 
Ad ogni punto $\vee$ di $S^{\prime}$ è associato un numero non negativo, la norma $\|v\|$ di $v$, nulla se e solo se $v=0$ e tale che, per ogni coppia $v^{\prime}, v^{\prime \prime}$, valga la limitazione:

$$
\left\|v^{\prime}-v^{\prime \prime}\right\| \leq\left\|v^{\prime}\right\|+\left\|v^{\prime \prime}\right\| \text {. }
$$

Infine, supposto che $\gamma(x)$ sia un punto di $S^{\prime}$ dipendente da una variabile reale $x$ e definito il

$$
\lim _{x \rightarrow x_{0}} v(x)=v_{0} \quad \text { quando } \quad \lim _{x \rightarrow x_{0}}\left\|v(x)-v_{0}\right\|=0
$$

è ammessa la validita del criterio di Botzano-CadCHy.

Per ogni insieme $E$ di $\mathscr{F}$, indicheremo con $m(E)$ la variazione totale della funzione $\mu(e)$ su $E$, cioè l'estremo superiore (finito, per ipotesi) dell'insieme numerico descritto dalle somme

$$
\left\|\mu\left(e_{1}\right)\right\|+\left\|\mu\left(e_{2}\right)\right\|+\ldots+\left\|\mu\left(e_{n}\right)\right\|,
$$

al variare comunque della decomposizione:

$$
E=e_{1}+e_{2}+. .+\stackrel{\circ}{+} e_{n} \quad\left(e_{i} \in \mathscr{F} ; i=1,2, \ldots, n ; n \text { intero posit. arbitr. }\right) .
$$

4. Diciamo chè un insieme, $I$, dello spazio metrico e separabile $S$, ̀̀ lebesghiano (rispetto alla massa $\mu(e)$ ) se, comunque si assegni un numero $\varepsilon>0$, è possibile costruire un insieme chiuso $C_{\varepsilon}$ contenuto in $I$ e un insieme aperto $A_{\varepsilon}$ contenente $I$, in modo che risulti $m\left(A_{\varepsilon}-O_{\varepsilon}\right)<\varepsilon$.

Con questa definizione è possibile ripercorrere, passo passo, buona parte della teoria di Pıoone relativa agli insiemi lebesghiani (Cfr. PV pag. 111 e segg.), sia pure con qualche leggera variante ch'è. molto fácile rilevare. Si comincerà col dimostrare:

che è lebesghiano il complementare, rispetto ad $S$, d'un insieme lebesghiano;

che sono lebesghiani gli insiemi chiusi e quelli aperti ( $\left.{ }^{(}\right)$;

che condizione necessaria e sufficiente affinchè un insieme $I$ sia lebesghiano è che, comunque si prefissi un numero $\varepsilon>0$, si possa costruire un insieme chiuso $C_{\varepsilon}$ e un insieme aperto $A_{\varepsilon}$, in modo che risulti

$$
C_{\varepsilon} \subset I \subset C_{\varepsilon}+A_{\hat{\varepsilon}}, \quad m\left(A_{\varepsilon}\right)<\varepsilon ;
$$

che il prodotto e la somma d'una succossione d'insiemi lebesghiani, sono anch' essi insiemi lebesghiani;

che la differenza di due insiemi lebesghiani ̀̀ un insieme lebesghiano.

(8) La dimostrazione PV p. 112 vale se $S$ si suppone euclideo (a un numero quaIunque di dimensioni). Nel caso più generale qui contemplato, una semplice dimostrazione può dedursi dal teorema: ogni insieme chíuso di uno spazio metrico e separabile è il limite di una successione non erescente d'insiemi aperti (Cfr. FIthera, loc. cit,, p. 59). 
Dopo cio si prolunghera la definizione della massa $\mu(e)$ sulla famiglia $\mathcal{S}_{k}$ degli insiemi e lebesghiani (famiglia contenente la $\mathscr{F}$ e completamente additiva al pari della $\mathfrak{F}$ ). semplicemente per passaggio a limite a partire dagli insiemi aperti che contengono $e$ oppure (cio ch'ó lo stesso) dagli insiemi ohinsi che sono contenuti in $e$. $\mathrm{E}$ analogamente si prolungherd la definizione della variazione totale $m(e)$. Si dovrì osservare però che la funzione reale m(e) assumeri sempre valori finiti (sia l'insieme $e$ limitato o no) $1^{9}$ ).

Infine si potrì dimostrare che $\mu(e)$ ed $m(e)$ sono completamente additive su $S_{\mu}$ ecc.

5. Diciamo che una funzione $f(P)$ assumente valori appartenenti allo spazio topologico $S^{\prime \prime}$. ̀े quasi continua (rispetto alla massa $\mu(e)$ ) in un insieme $I \mathrm{di} S$, se $I \in \AA_{p^{2}}$ e se, comunque si assegni il numero $\varepsilon>0$, $\partial$ possibile costruire un insieme $C_{\varepsilon} \subset I$, tale che in esso la $f(P)$ risulti continua $\theta$ sia

$$
m\left(I-C_{\varepsilon}\right)<\varepsilon .
$$

Sussistono le seguenti proposizioni $\left({ }^{10}\right)$.

Una lunzione quasi continua in un insicme lebesghiano $I$, è tale anche in ogni insieme lebesghiano $U \subset I$.

Condizione necessaria e sufficiente affinchè una funzione sia quasi continua in un insieme lebesghiano non limitato, è eh' essa sia tale in ogni por* zione lebesghiana limitata di quell'insieme.

Una funzione quası continua in nn insieme lebesghiano $I$, e definita in (quisi tutto $I$ : cioe l'eventmale insieme $\mathcal{Q}$ dei punti di $I$ nei quali essa non i definita, \& lebesghiano e tale che $m(\mathcal{Q C})=0$.

Una funzione che sia quasi continua in un insieme $I-\mathscr{T}$, essendo $\mathscr{\mathscr { C }}$ lebesghiano, contenuto in $I$ e tale che $m(\mathscr{Q C})=0$, è quasi continua anche in $I$.

Ena ennzione che sia quasi continna in ogni insieme della successione $I_{1}, I_{2}, \ldots, I_{n}, \ldots$, e tale anche negli insiemi

$$
\prod_{n}^{1, \infty} I_{n}, \quad \stackrel{1, \infty}{\Sigma} I_{n}, \quad \lim _{n \rightarrow \infty}^{\prime} I_{n}, \quad \lim _{n \rightarrow \infty}^{\prime \prime} I_{n} .
$$

6. La teoria delle successioni di funzioni quasi continue (nella trattazione, cosi elegante e feconda di risultati, esposta da PICONE) non sembra a questo punto potersi estendere senza introdurre l'ipotesi, più restrittiva, the lo spazio $S^{\prime \prime}$ sia metrico e completo. In tale ipotesi ci proponiamo di dimostrare la seguente proposizione (11), che sembra all uopo assolutamente fondamentale.

(P) Cfr. Ia nota, (19).

("9) Per le dimostrazioni clr. 1'V, np. 124 e segg.

(ii) Amaloga, in certo modo, alla I di PV, p. 129, $\$ 45$. 
Se $f(P)$ e $\varphi(P)$ sono due funzioni quasi continue in uno stesso insieme $I$ ed a è una costante positiva arbitraria, è lebesghiano l'insieme $I_{a}=I[\overline{f \varphi} \geq a]$, formato dai punti $P$ di $I$ nei quali la distanza $f \varphi \grave{e} \geq a$.

Infatti, in virtù dell'ipotesi, prefissato comunque un numero $\varepsilon>0$, ஓे possibile costruire due insiemi chiusi $C_{\sharp}^{\prime}, C_{\varepsilon}^{\prime \prime}$ e due insiemi aperti $A_{:}^{\prime}, A_{\varepsilon}^{\prime \prime}$ tali che

$$
C_{\varepsilon}^{\prime} \subset I \subset C_{\varepsilon}^{\prime}+A_{\varepsilon}^{\prime}, \quad C_{\varepsilon}^{\prime \prime} \subset I \subset C_{\varepsilon}^{\prime \prime}+A_{\varepsilon}^{\prime \prime}, \quad m\left(A_{\varepsilon}{ }^{\prime}\right)<\varepsilon_{2}^{\varepsilon}, \quad m\left(A_{\varepsilon}^{\prime \prime}\right)<\frac{\varepsilon}{2}
$$

e che $f(P)$ sia continua in $C_{g}^{\prime}, \varphi(P)$ sia continua in $C_{\xi}^{\prime \prime}$. Nell'insieme chiuso $C_{\varepsilon}=C_{\varepsilon}^{\prime} C_{\varepsilon}^{\prime \prime}$ risulteranno continue sia $f(P)$ che $\varphi(P)$, mentre per l'insieme aperto $A_{\varepsilon}=A_{\varepsilon}^{\prime}+A_{\varepsilon}^{\prime \prime}$ si avrà $m\left(A_{\varepsilon}\right)<\varepsilon$. Il prodotto $I_{a} C_{z}^{\prime}$, come luogo dei punti dell'insieme chiuso $C_{\varepsilon}$ in cui la funzione continua $\delta(P)=f \varphi \dot{\theta} \geq a$, è un insiene chiuso e pertanto, avendosi

$$
C_{\varepsilon} \subset I \subset C_{\varepsilon}+A_{\varepsilon}, \quad I_{a} C_{\S} \subset I_{a} \subset I_{a} O_{z}+A_{\varepsilon}
$$

si deduce la tesi enunciata.

Analoganente si dimostra che sono lebesghiani gli insiemi $I|\widetilde{f} \leq a|$, $I\left[\tilde{f}_{\varphi}=a\right]$. Saranno percio lebesghiani anche gli insiemi

$$
I[f \varphi>a]=I|\bar{\varphi} \bar{\varphi} \geq a|-I|f \varphi=a|,
$$

$I \mid \bar{f} \varphi<a]$ e (qualunque sia la costante reale $b>a)$

$$
I[a \leq \overline{f \varphi} \leq b]=I[f \varphi \geq a] . I[f \varphi \leq b] \text {, ecc. }
$$

Dalla proposizione or ora dimostrata si può dedurre il teorema: se le funzioni della successione (1) sono tutte quasi continue in uno stesso insieme lebesghiano $I$, sono lebesghiani sia l'insieme $J$ dei punti di $I$ in cui la (1) non converge, sia l'insieme $I-J$ dei punti in cui la (1) converge; il teorema di SeverinI-EgoroFF: se la successione (1) di funzioni tutte quasi continue in uno stesso insieme $I$, è quasi ovanque convergente in $I$, comunque si assegni un $\varepsilon>0$ eे possibile costruire un insieme lebesghiano $H_{\varepsilon} \subset I$, tale che $m\left(H_{\varepsilon}\right)<\varepsilon$ e che la successione (1) eonverga uniformemente in $I-H_{s} ; \theta$ da ultimo il teorema che abbiamo enunciato alle fine del n. $1\left({ }^{12}\right)$.

Passeremo ora all' argomento propostoci al n. 2.

(12) Le dimostrazioni sono in tutto identiche a quelle di 1 V V, pp. 134-139, ove soltanto si sostituiseano $i$ valori assoluti $\left|f_{n u}(P)-f_{n}(P)\right|$ con lo distanze $\overline{f_{m}(P) f_{n}(P)}$. 


\section{Studio di una successione di funzioni, nelle condizioni piú generali.}

7. In questo numero dimostreremo due proposizioni preliminari, nelle quali potremo supporre di nuovo (come fu fatto prima del n. 6) che lo spazio $S^{\prime \prime}$ (dei valori assunti dalle funzioni quasi continue considerate) sia semplicemente topologico. Ferme dorranno però restare le ipotesi fatte sui due spazi nei quali s'intendono variare rispettivamente il punto $P$ e la massa $\mu(e)$, ciò̀: $S$ metrico e separabile (n. 4), $S^{\prime}$ additivo (nel senso del n. 3 ).

LEMMa I. - Sia $f(P)$ una funzione quasi continua nell' insieme I e sia $\Gamma$ un qualunque insieme chiuso di $S^{\prime \prime}$. L'insieme

$$
I_{\Gamma}=I[f(P) \in \Gamma]
$$

formato da tulti $i$ punti $P$ di I nei quali $f(P)$ aqpartiene a $\Gamma$, è lebesghiano $\left({ }^{13}\right)$

Infatti, prefissato ad arbitrio un numero $\varepsilon>0$, è possibile costruire un. insieme $C_{\varepsilon}$ chiuso e di continuità per la $f(P)$, e un insieme aperto $A_{\varepsilon}$, in modo che risulti

$$
C_{\varepsilon} \subset I \subset C_{\xi}+A_{\varepsilon}, \quad m\left(A_{\varepsilon}\right)<\varepsilon .
$$

Il prodotto $I_{\Gamma} C_{\varepsilon}$ (come luogo dei punti dell'insieme chiuso $C_{\varepsilon}$, nei quali la funzione continua $f(P)$ assume valori appartenenti all' insieme chiuso $\Gamma$ ) è un insieme chiuso $\left({ }^{14}\right)$, donde segue immediatamente la tesi, osservando che

$$
I_{\Gamma} C_{\varepsilon} \subset I_{\Gamma} \subset I_{\Gamma} C_{\varepsilon}+A_{\varepsilon} .
$$

Lemma II. - Se le funzioni $f_{n}(P)$ della successione (1) sono tutte quasi continue in $I$, e se $\Gamma$ è un qualunque insieme chiuso di $S^{\prime \prime}$, l' insieme $\Gamma^{\prime}$ dei punti di $I$ in ciascuno dei quali infinite funzioni $f,(P)$ assumono valori appar. tenenti a $\Gamma$, ̀̀ lebesghiano.

Infatti, posto

$$
I_{n}=I\left[f_{n}(P) \in \Gamma\right] \quad(n=1,2, \ldots),
$$

risulta

$$
\Gamma^{\prime}=\lim _{n \rightarrow \infty}^{\prime \prime} 1_{n}
$$

Essendo dunque ciascun insieme $I_{n}$ lebesghiano in virtù del lemma $I$, anche $\Gamma^{\prime}$ è lebesghiano (v. n. 5) c. d. d.

(15) Questa proposizione offre chiara analogia con quella che abbiamo enunciata e dimostrata al n. 6.

(14) Cfr. M. Proone e G. Fronera, Trattato di Analisi Matematica (Roma, Tumminelli, 1954) vol. I, p. $166, X$. 
8. Veniamo da ultimo alla dimostrazione del teorema che abbiamo enunciato al n. 2, supponendo ormai che lo spazio $S^{\prime \prime}$ sia metrico, compatto e separabile.

Per l'ipotesi della compattezza di $S^{\prime \prime}$, a prescindere al piu da un insieme lebesghiano $\mathscr{K}$ tale che $m(\mathscr{V})=0$ (formato dagli eventuali punti di $I$ in cui al più un numero finito di funzioni $f_{n}(P)$ sono definite), in ogni punto $P$ di $I$ la successione (1) ammette almeno un valor limite: indichiamo con $\mathfrak{Q}_{P}$, per ogni punto $P$ di $I-\mathscr{N}$, l'insieme chiuso $\subset S^{\prime \prime}$ formato da tutti i valori limiti della successione (1), indi poniamo

$$
H=\underset{P \varepsilon I-\mathscr{Q}}{\Sigma} \mathscr{Q}_{P}
$$

Sia $B \equiv\left(b_{1}, b_{2}, \ldots\right)$ la base di $S^{\prime \prime}$ e siano $Q_{r}(\rho)(r=1,2, \ldots)$ i domini, di $S^{\prime \prime}$, circolari e di raggio $\rho$, i cui centri sono rispettivamente nei punti $b,$.

Diciamo $R_{14}$ il primo dei domini $Q_{r}(1)(r=1,2, \ldots)$, sia $Q_{i_{1}}(1)$, che contiene nel proprio interno almeno un punto di $H$, ed $I_{11}$ l'insieme (lebesghiano, in virtù del lemma II) formato dai punti $P$ di $I-\mathscr{N}$, in ciascuno dei quali infinite funzioni $f_{n}(P)$ assumono valori appartenenti ad $R_{11}$.

In virtù della compattezza di $S^{\prime \prime}$, se $P \in I_{11}, \mathscr{U}_{P} R_{11}$ non è vooto; invece, se $P \in I-\mathscr{T}-I_{11}$, si può evidentemente dire soltanto che $\mathfrak{L}_{P}$ non possiede punti interni ad $R_{11}$.

Supponiamo cha sia $I-\mathscr{N} \neq I_{44}$. Allora, percorrendo la successione $\left\{Q_{i_{1}+r}(1)\right\} \quad(r=1,2, \ldots)$, si troverà certamente un primo dominio $R_{42}$, sia $Q_{i_{2}}(1)$, soddisfacente alle condizioni:

a) $R_{12}$ contiene, nel proprio interno, almeno un punto di $H$ non interno ad $R_{11}$;

b) l'insieme lebesghiano ( $\left.{ }^{15}\right) I_{12}$ formato dai punti $P$ di $I-\mathscr{T}-I_{14}$, in ciascano dei quali infinite funzioni $f_{n}(P)$ assumono valori appartenenti ad $R_{12}$, non è vuoto.

Così proseguiamo ordinatamente. Supponiamo d'aver determinato un certo gruppo di $p$ domini circolari $R_{11}, R_{12}, \ldots, R_{1 p}$ (con $p \geq 1$ ) e, corrispondentemente, un certo gruppo di $p$ insiemi lebesghiani $I_{11}, I_{12}, \ldots, I_{1 p}$, due a due disgiunti, tali che sia

$$
I-\mathscr{O} \neq I_{11} \stackrel{\circ}{+} I_{12}+\ldots \stackrel{\circ}{+} I_{1 p}
$$

Percorrendo la successione $\left\{Q_{i_{p}+r}(1)\right\}(r=1,2, \ldots)$, si troverà certamente un primo dominio $R_{1, p+1}$, sia $Q_{i_{p+1}}(\mathrm{l})$, soddisfacente alle condizioni :

a) $R_{1, p+1}$ contiene, nel proprio interno, almeno un punto di $H$ non interno ad $R_{11}+R_{12}+\ldots+R_{1 p}$

('5) $I-\mathscr{V}-I_{11}$ ̀̀ lebesghiano come differenza d'insiemi lebesghiani. $I_{12}$ è dunque lebesghiano in virtù del lemma II. 
b) l'insieme lebesghiano $I_{1, p+1}$ formato dai punti $P$ di $I-\mathscr{V}-$ $-\left(I_{11}+I_{12}+\ldots+I_{1 p}\right)$, in ciascuno dei quali infinite funzioni $f_{n}(P)$ assumono valori appartenenti ad $R_{1, p+1}$, non è vuoto.

Se il procedimento indicato non ha termine, cioè se non si perviene ad un intero $p>0$ tale che sia $I-\mathscr{G}=I_{11} \stackrel{\circ}{+} I_{12}+\ldots \stackrel{\circ}{+} I_{1 p}$, si verrà così a determinare una successione d'insiemi $I_{s p}(p=1,2, \ldots)$ tale che, come subito si riconosce,

$$
I-\mathscr{K}=I_{11} \stackrel{\circ}{+} I_{12} \stackrel{\circ}{+}+. \stackrel{\circ}{+} I_{1 p} \stackrel{\circ}{+} \ldots
$$

Definiamo, in ogni caso, nell'insieme $I-\mathscr{T}$, la funzione quasi continua

$$
\left.F_{1}(P)=b_{i_{p}} \text { (centro di } R_{1 p}\right) \text {, per } P \in I_{1 p} \quad(p=1,2, \ldots) \text {. }
$$

9. Immaginando ora di fissare uno qualunque degli indici $p \geq 1$, diciamo $R_{1}^{1 p}$ il primo dei domini $Q .\left(\frac{1}{2}\right)(r=1,2, \ldots)$, sia $Q_{f_{1}}\left(\begin{array}{l}1 \\ 2\end{array}\right)$, ohe contiene nel proprio interno almeno un punto del prodotto $H R_{1 p}$, ed $I_{1}^{1 p}$ l'insieme lebesghiano formato dai punti $P$ di $I_{i p}$, in ciaseuno dei quali infinite funzioni $f_{,}(P)$ assumono valori appartenenti ad $R_{1}^{1 p}$

Se è $I_{1 p} \neq I_{1}^{1 p}$, si riconosce subito (analogamente à quanto sopra, v. n. 8) che, percorrendo la successione $\left\{Q_{j_{1}+},\left(\frac{1}{2}\right)\right\}(r=1,2, \ldots)$, si dovrà trovare un primo dominio $R_{2}^{1 p}$, sia $Q_{j_{2}}\left(\frac{1}{2}\right)$, soddisfacente alle condizioni :

a) $R_{2}^{l p}$ contiene, nel proprio interno, almeno un punto di $H R_{1 p}$ non interno ad $R_{1}^{1 p}$;

b) l'insieme lebesghiano $I_{2}^{1 p}$ formato dai punti $P$ di $I_{1 p}-I_{1}^{1 p}$, in cia. scuno dei quali infinite funzioni $f_{n}(P)$ assumono valori appartenenti ad $R_{2}^{\prime p}$, non è vuoto.

Cosi proseguiamo ordinatamente. Supponiamo d'aver determinato un certo gruppo di $q$ domini circolari $R_{1}^{\mathbb{t p}}, R_{2}^{1 p}, \ldots, R_{q}^{1 p}$ (con $q \geq 1$ ) e, corrispondente. mente, un certo gruppo di $q$ insiemi lebesghiani $I_{1}^{1 p}, I_{2}^{1 p}, \ldots, I_{q}^{1 p}$, due a due disgiunti, tali che sia $I_{1 p} \neq I_{1}^{1}{ }^{\circ} \ldots+\ldots+I_{q}^{1 p}$. Percorrendo la successione $\left\{Q_{j_{q}+v}\left(\frac{1}{2}\right)\right\}$ $(r=1,2, \ldots)$ si troverà certamente un primo dominio $R_{q+1}^{1 p}$, sia $Q_{j_{q+1}}\left(\frac{1}{2}\right)$, sod. disfacente alle condizioni :

a) $R_{q+1}^{1 p}$ contiene, nel proprio interno, almeno un punto di $H R_{i p}$ non interno ad $R_{1}^{1 p}+R_{2}^{1 p}+\ldots+R_{q}^{1 p}$

b) l'insieme lebesghiamo $I_{q+1}^{1 p}$ formato dai punti $P$ di $I_{1 p}-\left(I_{1}^{1 p} \stackrel{\circ}{+} I_{2}^{1 p} \stackrel{\circ}{+} \ldots\right.$ $\left.\ldots+I_{q}^{1 p}\right)$, in ciascuno dei quali infinite funzioni $f_{n}(P)$ assumono valori appartenenti ad $R_{q+1}^{1 p}$, non è vuoto. 
Se il procedimento indicato non ha termine, cioè se non si perviene ad un intero positivo $q$ tale che sia

$$
I_{1 p}=I_{1}^{1 p}+I_{2}^{1 p}+\ldots \stackrel{\circ}{+} I_{q}^{1 p},
$$

si verrà cosí a determinare una sucoessione d'insiemi $I_{q}^{1 p}(q=1,2, \ldots)$ tale che

$$
I_{1 p}=I_{1}^{1 p} \stackrel{\circ}{+} I_{2}^{1 p} \stackrel{\circ}{+} \ldots \stackrel{\circ}{+} I_{q}^{1 p} \stackrel{\circ}{+} \ldots .
$$

Disponiamo la successione doppia $\left\{I_{q}^{1 p} \mid(p=1,2, \ldots ; q=1,2, \ldots)\right.$ in una successione semplice che indichiamo con $\left\{I_{2 p}\right\}(p=1,2, \ldots)\left({ }^{16}\right)$ e, corrispondentemente, la successione doppia $\left|R_{q}^{1 p}\right|(p=1,2, \ldots ; q=1,2, \ldots)$ nella suc. cessione semplice che indichiamo con $\left\{R_{2 p}\right\}(p=1,2, \ldots)$, e definiamo in $I-\mathscr{T}$ la funzione quasi continua

$$
F_{2}(P)=b_{j_{p}}\left(\text { centro di } R_{i p}\right) \text {, per } P \in I_{2 p} \quad(p=1,2, \ldots) .
$$

10. Cosi proseguiamo ordinatamente e indefinitamente.

Supponiamo d'esser pervenuti alla costruzione delle successioni

$$
\left\{I_{t p}\right\},\left\{I_{2 p}\right\}, \ldots,\left\{I_{s p}\right\} \quad(p=1,2, \ldots)
$$

(per un certo intero $s \geq 1$ ) d'insiemi tutti lebesghiani (17). Immaginando di fissare uno qualunque degli indici $p \geq 1$, diciamo $R_{1}^{s p}$ il primo dei domini $Q .\left(\frac{1}{2^{s}}\right)(r=1,2, \ldots)$, sia $Q_{n_{1}}\left(\frac{1}{2^{s}}\right)$, che contiene, nel proprio interno, almeno un punto di $H R_{s p}$, ed $I_{1}^{s p}$ l'insieme lebesghiano formato dai punti $P$ di $I_{s p}$, in ciascuno dei quali infinite funzioni $f_{n}(P)$ assumono-valori appartenenti ad $R_{1}^{s p}$. E, dopo ciò, ripetiamo parola per parola tutto quanto abbiamo detto al n. 9, con l'unica differenza di sostituire sempre la coppia d'indici $1 p$ (in ogni simbolo in cui questa compare) con la coppia sp, e di scegliere i domini circolari dalla successione $\left\{Q_{r}\left(\frac{1}{2^{s}}\right)\right\}(r=1,2, \ldots)$ anzichè dalla successione $\left\{Q_{r}\left(\frac{1}{2}\right)\right\}$; infine definiamo la funziono quasi continua

$$
\left.F_{s+1}(P)=b_{h_{p}} \text { (centro di } R_{s+1, p}\right), \text { per } P \in I_{s+1, p} \quad(p=1,2, \ldots) .
$$

Affermiamo che la successione $\left\{F_{s}(P)\right\}(s=1,2, \ldots)$ è convergente in quasi tutto $I$, precisamente in tutto $I-\mathscr{N}$. Infatti se $P_{0} \in I-\mathscr{K}$, è definita una ben determinata successione d'insiemi'

$$
I_{1 p_{1}} \supset I_{2 p_{s}} \supset \ldots \supset I_{s p_{s}} \supset \ldots
$$

(16) Si osservi che gli insiomi di tale sucessione semplice risultano due a due disgiunti. (17) Ognuna di queste successioni è formata d'insiemi due a due disgiunti (Cfr. la nota precedente). 
tutti contenenti $P_{0}$. Indicati allora con

$$
b_{i_{1}}, b_{j_{p_{2}}}, \ldots, b_{l_{p_{s}}}, \ldots
$$

i centri dei corrispondenti domini

$$
R_{1 p_{1}}, R_{2 p_{2}}, \ldots, R_{8 p_{s}}, \ldots
$$

si riconosce subito che, in virtù della completezza di $S^{\prime \prime}$, esiste il limite, unico e ben determinato, della successione (2). La funzione $f(P)$ che, in $P_{0}$, è uguale a siffatto limite, risponde alla tesi del teorema. Infatti si ha, in primo luogo,

$$
\lim _{s \rightarrow \infty} F_{s}(P)=f(P)
$$

in tutto $I-\mathscr{T}$, e perciò $f(P)$ ஓे quasi continua in $I$. In secondo luogo, in virtù della costruzione indicata, la successione $\left\{f_{n}\left(P_{0}\right)\right\}$ possiede infiniti termini in $R_{s p_{s}}$ (qualunque sia $s=1,2, \ldots$ ), cioè termini la cui distanza da $b_{l_{p_{s}}}$ (centro di $R_{s p_{s}}$ ) non supera $\frac{1}{2^{s-1}}$ : tale successione possiede dunque infiniti termini anche in ogni intorno del punto indicato con $f\left(P_{0}\right)$ e con eio rimane dimostrato ohe $f\left(P_{n}\right)$ ¿े uno dei valori limiti della succeśsione stessa, c. d. d.

11. Pù essere interessante ricercare se e come il teorema che abbiamo dimostrato, trovi applicazione al caso che i valori assunti dalle funzioni della successione (1) appartengano ad uno spazio $S^{\prime \prime}$ che sia bensi metrico, com. pleto e separabile, ma non precisamente compatto: anzi, come accade per uno spazio euclideo a un numero qualunque di dimensioni, goda della pro. prietà che siano compatti soltanto $i$ suoi domini limitati.

Una tale ricerca può essere fatta, nel modo più semplice, cominciando col predisporre, in $S^{\prime \prime}$, una successione di domini circolari concentrici $\Gamma_{m}$ $(m=1,2, \ldots)$, aventi raggi uguali ai propri indici. In corrispondenza di ogni valore dell'indice $m$, diciamo $G_{m}$ l'insieme, lebesghiano in virtù del lemma II, formato dai punti $P$ di $I-\mathscr{N}$, in ciascuno dei quali infiniti termini della successione (1) cadono in $\Gamma_{m}$. E evidente che $G_{m}$ contiene tutti i punti $P$ di $I-\mathscr{X}$ tali che $\mathcal{Q R}_{P}$ abbia almeno un punto interno a $\Gamma_{m}$.

Poichè $G_{1} \subset G_{2} \subset \ldots \subset G_{m} \subset \ldots$, l'insieme $G=\lim _{m \rightarrow \infty} G_{m}$ è lebesghiano e comprende tutti e soli i punti $P$ di $I-\mathscr{N}$ in ciascuno dei quali la (1) possiede almeno un valore limite. D'altra parte, se consideriamo le funzioni della (1) come definite in $G_{1}$, potremo ripetere tutti i ragionamenti dei $\mathrm{nn}$. 8-10 soltanto con qualche lieve alterazione di dettaglio: la più rilevante delle quali consiste nel sostituire alla considerazione dell' intero spazio $S^{\prime \prime}$, quella del dominio $\Gamma_{1}$ (assunto come spazio, compatto, dei valori di infinite funzioni $f_{n}(P)$ ). 
Si giungerd cosi a costruire una ben determinata funzione $f(P)$, quasi continua in $G_{1}$, che in ogni punto $P$ di $G_{1}$ è uguale a uno dei valori limiti della (1). Analogamente potra ragionarsi successivamente sugli insiemi $G_{n+1}-G_{n}$ $(n=1,2, \ldots)$, assumendo come corrispondenti spazi, compatti, i domini $\Gamma_{n+1}$. Si può dunque concludere col seguente teorema $\left({ }^{18}\right)$ :

nelle ipotesi fatte per la successione (1) nell' insieme I, la parte $G$ di I formata dai punti $P$ nei quali la successione ammette almeno un valore limite, è lebesghiana. $\grave{E}$ possibile costrwire una funzione $f(P)$ quasi continua in $G$, che, in ogni punto $P$ di $G$, è uguale ad uno dei valori limiti della successione $\left({ }^{19}\right)$.

(18) Ofr. il teor. II di PV, p. 141.

(19) Un'altra generalizzazione della teoria svolta ê possibile: quella che s'ottiene supponendo che la massa $\mu(e)$ sia a variazione finita negli insiemi limitati $e$ di $\mathfrak{F}$ (má possa non esser tale su certi insiemi illimitati di?f). Lasciamo al lettore $i$ facili ritocchi necessari in qualche particolare dettaglio, avvertendo soltanto che inulla cambia nei risultati finali di questo lavoro. 